OCEANS'15 MTS/IEEE Washington Proceedings. Systems and Observatories 2

Pages 1-8

http://dx.doi.org/10.23919/OCEANS.2015.7404554

http://archimer.ifremer.fr/doc/00295/40665/

(C) 2015 IEEE

\title{
Harmonization in the Joint European Research Infrastructure Network for Coastal Observatories -JERICO
}

\author{
Petihakis George ${ }^{1,{ }^{*}}$, Petersen Wilhelm ${ }^{2}$, Nair Rajesh ${ }^{3}$, Faimali Marco ${ }^{4}$, Pavanello Giovanni ${ }^{4}$, \\ Boukerma Kada ${ }^{5}$, Delauney Laurent ${ }^{5}$, Puillat Ingrid ${ }^{5}$, Farcy Patrick ${ }^{5}$, Greenwood Naomi ${ }^{6}$
}

\footnotetext{
${ }^{1}$ Hellenic Centre for Marine Research - HCMR Heraklion Crete, Greece

${ }^{2}$ Helmholtz Zentrum Geesthacht - HZG Geesthacht, Germany

3 Istituto Nazionale di Oceanografia e di Geofisica Sperimentale - OGS Sgonico (Trieste), Italy

${ }^{4}$ Consiglio Nazionale delle Ricerche - CNR ISMAR Genova, Italy

${ }^{5}$ Institut Français de Recherche pour l'Exploitation de la Mer - IFREMER Plouzané, Brest, France

${ }^{6}$ Cefas Lowestoft, UK

*Corresponding author : George Petihakis, email address : gpetihakis@hcmr.gr
}

\begin{abstract}
:
The JERICO European research infrastructure $(R I)$ is integrating diverse platform types such as fixed buoys, piles, moorings, drifters, FerryBoxes, gliders, HF radars, coastal cable observatories and the associated technologies dedicated to observe and monitor coastal European seas. The first steps of setting up, coordination and harmonization were done during 2011 to 2015 in the framework of FP7JERICO (www.jerico-fp7.eu), a 4-year long infrastructure project co-funded by the European Commission with 27 partners from 17 European countries under the coordination of IFREMER. Next steps are driven in the H2020-JERICO-NEXT European project until 2019, involving 33 partners. The main objective of the JERICO consortium is to establish a Pan European approach for a European coastal marine observatory network. This is a dynamic activity going beyond a project's lifetime including continuous efforts towards harmonization in terms of design, operation, and maintenance, the evolution and extension of the current systems as well as the delivery of data and products to the users. Our scope here is to present the work done towards the harmonization of operation and maintenance methods, in FP7-JERICO and the next steps in JERICO-NEXT. As a starting point of harmonization assessment, the priority was given to the most pressing issues like calibration and biofouling, while it is the first time that a Best Practice report on all phases of the system from first installation to operation and maintenance is attempted adopting a platform based approach.
\end{abstract}

Keywords : coastal observatories, harmonization, biofouling, calibration, JERICO 


\section{Introduction}

The seas and oceans are an intrinsic part of the earth and climate systems. They cover $70 \%$ of our planet, provide $95 \%$ by volume of its biosphere, support more than $50 \%$ of global primary production and harbor an enormous diversity of life adapted to extremely broad-ranging environmental conditions. The oceans are a driver of our climate but are also affected by climate change and ocean acidification. They are under increasing pressure from human activities and pollution, and growing coastal populations. The combination of natural and human-induced changes taking place in our seas and oceans including, for example, rising temperatures, the melting of Arctic sea ice, ocean acidification, increasingly extreme weather events, transfer of non-indigenous marine species, changes in biodiversity and species distribution, and depletion of fisheries stocks, may have potentially profound impacts on our societies and economies in the medium-term. European research focused on the seas and oceans is of uttermost importance in order to address these challenges. Such research should deliver knowledge and tools enabling Europe to prepare for, and adapt to, these changes. Moreover the growth of new and existing industries such as marine renewable energy, marine biotechnology, fisheries and aquaculture and sustainable maritime transport must be supported by research and innovation, involving a range of actors to develop technologies and best practices in support of a thriving European maritime economy. 
A key issue is how society, in the coming decades, will tackle these threats and turn challenges into opportunities. The Rio Ocean declaration (16 June 2012) called for an "integrated approach addressing the interlinked issues of oceans, climate change, and security" and for countries to "Establish the scientific capacity for marine environmental assessment, monitoring, and prediction, including the implementation of the global ocean observing system". Routine and sustained ocean observations are crucial to further increase our understanding of the complex and vast oceanic environment and to supply scientific data and analyses sufficient to meet society's needs. In particular, for the coastal environment needs are even higher, as the natural variability is interlinked with human induced changes, and efforts to identify and distinguish the various components are crucial. Furthermore most economic activity is based at the coastal ocean.

Europe spends $€ 1.4$ billion p.a. for marine data collection: $€ 0.4$ billion for satellite data and $€ 1.0$ billion for in-situ observations, respectively. In the case of the latter, the traditional and expensive practice of vessel-based data gathering is progressively giving way to monitoring via "observatories" - complexes of distributed, autonomous, realtime sensor systems. Burgeoning technology and pressing societal needs will soon make such observatories the backbone of European marine observing activity because of their ability to provide copious quantities of diversified data over large areas at reasonable costs.

Around European coastal seas, the number of marine observing systems is quickly increasing under the pressure of both monitoring requirements and oceanographic research. Present demands for such systems include reliable, highquality and comprehensive observations, automated platforms and sensors systems, as well as autonomy over long time periods. In-situ data collected, combined with remote sensing and models output, contribute to detect, understand and forecast the most crucial coastal processes over extensive areas within the various national and regional marine environments.

Coastal observations are an important part of the marine research puzzle of activities and applications. However significant heterogeneity exists in Europe concerning technological design of observing systems, measured parameters, practices for maintenance and quality control, as well as quality standards for sensors and data exchange. Coastal observatories have been developed in Europe in a rather uncoordinated way. Usually based on national funding and priorities these observatories have very diverse design and architecture and have established very different practices for their operation and maintenance. For certain subsystems (e.g. FerryBox) past EU projects have established a network of operators through which experience and best practices have been shared [1] but this is not the case for other observing platforms, and certainly not for integrated coastal observatories.

FP7-JERICO ("towards a Joint European Research Infrastructure network for Coastal Observatories", www.jericofp7.eu) is a 4-year long infrastructure project co-funded by the European Commission, including 27 partners from 17 European countries, under the coordination of IFREMER. The
Project proposes a Pan European approach for a European coastal marine observatory network, integrating infrastructure and technologies. Considering the importance of observing systems and the substantial investment made until now, an important task of the Project was to describe best practices in all phases of the system (pre-deployment test, maintenance, calibration etc.), to adopt common methodologies and protocols and to move towards the harmonization of equipment, which will help in reducing maintenance and calibration costs. These efforts and outcomes are described and analyzed.

\section{HARMONISATION OF OPERATION AND MAINTENANCE METHODS}

The past decade has seen a major effort towards the development of marine observations targeted at a better understanding of biogeochemical cycling and ecosystem services. Methods for ocean observation are constantly evolving and innovation is an essential driver for science and engineering excellence and technological advancement. New smart sensors, techniques and platforms are emerging with the aim of providing automated solutions for multidisciplinary marine monitoring. For in situ ocean observation, improvement of sensitivity, accuracy, stability, resistance to oceanic conditions and depth rating are all key factors for ensuring high quality, affordable data. During the $90 \mathrm{~s}$, an increased interest in ocean observation led to a huge technological advancement in automated sensors for monitoring physical variables such as temperature, salinity and currents. In the last years much work was focused on minimizing power requirements and reducing the size of sensors towards miniaturized lab-on-a-chip micro sensors, able to minimize the payload and enable multiparametric observation from single platforms such as gliders and drifting buoys. Operation and maintenance activities are probably the most crucial elements in the life cycle of a research infrastructure and in some cases even more demanding than the design and construction of the infrastructure itself $[2,3]$. Their successful implementation guarantees the good performance of the infrastructure and the protection of the investment.

\section{A. Biofouling}

The unwanted settlement and growth of organisms (biofouling) on man-made structures immersed in the sea is a common problem across observing systems, even though there is a significant gradient of magnitude in European Seas - north to south and west to east. Additionally, each sensor has its own characteristics and requirements. Although during the last few years significant progress has been achieved towards the minimization of bio fouling on optical measurements with the introduction of copper shutters, wipers or scrapers and localized chlorination, the problem still exists [4]. Concerning biofouling, major tasks in JERICO were to:

1) Describe all the different methods used by project partners, with reference to the cost (implementation, maintenance) and adaptability (different sensors and areas); 
A survey was carried out within the JERICO community, i.e. amongst the member of the project consortium. Answers were provided by 19 partners for 23 platforms and 54 sensors/sensors systems. The questionnaire was organized in 2 parts: Part A aimed at collecting information on the perception of the problem and on the most common approaches used to cope with biofouling, and Part B focused to collect information on specific sensors/systems in use.

Summarizing, most partners take into account biofouling prevention when choosing a sensor among different providers, and to avoid biofouling growth most of their infrastructures/installations use mechanical devices and uncontrolled biocide generation system, choice that can be attributed to economic and reliability aspects. Regarding uncontrolled biocide generation, many partners point toward "smarter" solutions, like controlled biocide generation, feeling confirmed by the necessity expressed by partners of having antifouling systems with a lower environmental impact. Most partners consider advantageous also closed-path systems, which is, conversely, a solution rarely applied by sensors producers. However, nearly all partners think that biofoulingrelated issues are really taken into account by sensor developers, highlighting that there is a growing concern by manufacturers about biofouling prevention, in particular for long term deployments. In parallel, it seems that there is still a large space for the growth of this market. When biofouling affects the data provided by sensors, for about $20 \%$ of sensors only it seems to be possible to distinguish between the effects attributable to a deterioration of the housing and those caused by the degradation of the sensing element. This underline the need of protecting the whole sensor.

All the partners are aware that biofouling represents a real problem in their observing activities, even if most of them indicate that it influences the money and the time spent for the mentioned activities only for $10 \%$ of the total. Annual expenses for antifouling systems in half cases is less than $1 \mathrm{~K} €$ (in more than $25 \%$ cases is less than $0.5 \mathrm{~K} €$ ). One third of partner's sensors do not have even any antifouling protection. The largest part of partners indicates that it is not aware of any recurring differences in the extensions/distributions of the various types of biofouling (biofilm/slime, hard-fouling, softfouling) related to the kind of sensor (physical, optical, chemical) or to the depth at which the sensor is placed. About one third of partners is not aware of any difference even in relation to the seasons. Moreover, just $60 \%$ of partners evaluate in situ biofouling pressure when deploying a biofouling prevention system. All these data suggest that biofouling phenomenon is not examined in depth before, during and after sensors deployment, even if partners are aware that it causes huge problems. Indeed, two third of partners confirm that biofouling influence the quality of the data collected by their sensors over time during a deployment, and questionnaire answers suggest that, on the sensors subjected to accuracy loss due to biofouling, partners apply antifouling protection. Half partners apply corrections to collected data, to compensate biofouling-related drifts. For about half of the sensors protected with antifouling systems, maintenance is expected to be performed at least every 6 months. On the other side, for about half of the sensors not protected with such systems, maintenance is expected to be performed at least every 3 months. This confirms both the awareness of partners for what concerns biofouling-related issues and the (partial, at least) efficacy of the applied antifouling technologies. However, onsite maintenance of sensors is very expensive and there is still a strong need of reducing the frequency of such operations implementing new and/or optimized techniques.

2) Share best practices and methodologies across the network with the aim towards a common approach;

Taking advantage of the consortium capacity, the partners designed and implemented a common biofouling experiment where selected sites representing different European marine environments acted as a test bed. ISMAR-CNR developed a special sampling system (Biofouling Monitoring Box - BMB) deployed by each partner in each sampling site. The BMB is a monitoring device specifically designed to provide substrates with spatial and structural heterogeneity that can simulate the complexity of the sensors and sensor housing/containers. Each partner was asked to immerse the system (BMB) close to a particular sensor, selected as the reference sensor, for this longterm (one year) study.

The settlement of marine organisms is influenced by complex interaction between different variables, including biotic and abiotic factors. The choice of settlement substratum is modulated by a series of abiotic factors that include environmental parameters and the physical nature of surfaces; these parameters also affect the biofilm growth that represents in turn an important biotic factor that influences larval settlement. Biofilm formation is strictly related to surface characteristics, and it is known to play a key role in larval settlement as happens, for example, in Amphibalanus amphitrite [5].

The experiments performed in different sampling areas showed, as might be expected, that the fouling community was very different among sites. After 3 months of exposure, Genoa was the site with the highest percentage of hard fouling, followed by ISMAR-Venice, IFREMER, HCMR. The fouling community observed in the Ifremer site was very different form the one observed in Genoa site. A great variety of taxa was mainly represented by organisms belonging to soft fouling.

Despite the differences in fouling composition, the light exposure played a key role in fouling community as it was observed in Genoa site where hard fouler preferred dark exposition.

As shown by our data, we investigated the role of some abiotic factors that deeply affected the settlement of a variety of macro- and microscopic marine organisms. Despite the differences in fouling composition among sampling sites, some factors played always a key role in the settlement of organisms. Such factors included the light availability and the nature of materials employed (metal, plastic and glass).

Our results contribute to show that organisms' settlement is a process affected by many chemical, physical and biological 
factors. These field experiments highlight how complex and numerous are the interactions among factors, that cannot mimicked in the laboratory. These mutual interactions play a key role during the settlement process, determining the variety and the heterogeneity of fouling community, which characterize the different sampling sites.

Comparing the data from the different sampling sites, we can learn more about the basic processes that determine the complex phenomenon of fouling colonization. Such studies are extremely important in order to prevent biofouling colonization and related issues, and these preliminary experiments move a first step toward this direction. Further joint research has to be carried out in this field in order to understand all the interactions involved and to obtain a better characterization of settlement behavior and fouling process.

3) To evaluate new antifouling methods used by the community external to JERICO and suggest possible future implementation.

The bibliographic review presented in the corresponding project deliverable showed that, in the field of biofouling protection for oceanographic sensors, just few innovations have seen the light during the last years. Among these, the most interesting and promising approach to developing novel sustainable solutions to biofouling is known as biomimetic design. This involves the study, characterization and transfer of natural antifouling mechanisms (surface structure and surface chemistry) utilized by aquatic organisms into artificial antifouling materials or solutions. From the application point of view, this biomimetic antifouling approach, based on physical and mechanical principles, seems to be particularly suitable in specialized markets such as the marine sensors one, thanks to the small surface area to be protected and the kind of materials utilized in the construction of these instruments. Currently, just a small number of lab/pilot applications of biomimetic surfaces exist, and it is quite hard to think that such technology will be used widely in the next years, mainly due to cost and scalingup related issues, even though they remain extremely promising.

On the other side, as came out from the survey performed among JERICO partners, there is a strong need for antifouling technologies which could make sensor deployment and maintenance (and, therefore, data acquisition) less expensive, guaranteeing the quality of collected data during long-term deployments. Such solutions should be reliable, cost-effective, and environmentally friendly and should require a reduced maintenance. All these in an extreme environment as the open ocean is a hard challenge, which requires both basic research on biology, material science, engineering, chemistry (and other fields) and, at the same time, a strong technology transfer to and collaboration with private companies.

\section{B. Calibration}

Reliable calibrations of instruments require wellestablished, documented procedures, specialized instrumentation, certified or recognized reference material (where these are available), dedicated laboratory facilities, trained personnel, and proven expertise. Although sensor calibration is absolutely crucial for good quality data, it is also a rather difficult task since different sensors have completely different requirements (time intervals) and methodologies.

There are two major problems; shipping sensors to manufacturers on regular basis which is neither convenient nor cost efficient and maintenance intervals that have to be planned according to the requirements of each sensor (need for double sets of sensors). Thus transport and calibration costs often have a major contribution on total running costs. Although there is significant experience among European research institutes on calibration methods, at present each lab works independently with no or very little connections with other labs. As described in the Description of the Work document, a major aim within JERICO was to:

- Standardize and harmonize various facilities across European networks,

- Share existing calibration facilities within the network, thus significantly reducing costs,

- Exchange and transfer know-how within the network through a series of workshops, seminars and staff exchange.

Operation and maintenance activities are probably the most crucial elements in the life cycle of a research infrastructure and in some cases even more demanding than the design and construction of the infrastructure itself. A sensor is only as good as its calibration, so a good sensor produces only poor results if the calibration is insufficient. Good sensors observations require both reliable sensor measurement methods and reliable calibration procedures. The successful implementation of operation and maintenance activities guarantees the good performance of the infrastructure and the protection of the investment. As it is the case for the observatories also the calibration infrastructure it quite diverse within Europe. Therefore, more work is needed to gather and combine information of relevant calibration issues.

The term calibration is defined as an operation that establishes a relation between the quantity values with measurement uncertainties provided by measurement standards and corresponding indications with associated measurements uncertainties and, in a second step, uses this information to establish a relation for obtaining a measurement result from an indication (JCGM, 2012). Sometimes, however, the word calibration is misused to describe the process of altering the performance of an instrument to ensure that the values it indicates are correct within specified limits (e.g. adjusting an instrument until its reading agrees with that of another instrument). Strictly this is adjustment - defined as the operation of bringing a measuring instrument into a state of performance suitable for its use - and not calibration, although the nature and magnitude of the adjustment is often determined by a pre-adjustment calibration, sometimes known as an as found calibration (NPL, 2014). 


\section{a) Calibration Best Practice recommendations}

From a series of common workshops and exercises during the project life cycle, partners defined a set of best practices:

- The proper calibration of dissolved oxygen sensors requires expertise, specialized equipment and procedures, dedicated staff, and most of all experience. If these resources are lacking in-house, it is better to send the sensors to the manufacturer for calibration or avail of an external provider of similar services.

- All the elements of the reference measuring systems must be maintained to within declared specifications by monitoring their performances regularly, adhering to recommended usage and upkeep practices, and scheduling servicing with a manufacturer immediately when laboratory quality assurance procedures indicate a developing problem.

- The dissolved oxygen reference measurements (e.g. Winkler analyses) should be regularly checked through Inter Laboratory Comparisons (ILC).

- All the information needed to understand the way reference measurement was obtained must be documented and stored to ensure traceability (equation used, parameters of the titrator, values of blanks, etc.).

- Sensors should be visually inspected prior to calibrating.

- The dissolved oxygen calibration facility should be allowed to settle at a calibration set-point for a sufficient period of time (an hour or more) before sampling is initiated. The stability of the bath should be continuously monitored during the sampling interval.

- The calibrated sensors should be checked at least at a few calibration set points prior to releasing them for duty.

- In principle, reference $\mathrm{O} 2$ measurements could be acquired using internal transfer standards (for example, a $\mathrm{O} 2$ sensor) in lieu of a analytical reference measurement, provided traceability to the appropriate primary standards has been established and the quality of readings are compatible with the degree of uncertainty required for calibrating.

- Marine O2 sensors require regular, often frequent, calibrations because their performances tend to vary over time and can be affected by the specific conditions of usage and storage. Sensor calibrations need to be verified at least once a year.

- Proper field maintenance is the key to successful calibrations. Poorly maintained instruments often need to be subjected to long and complicated procedures in order to restore them to a condition that would permit a proper calibration to be performed.

- In the case of modular O2 sensors, sensor calibrations must be performed, whenever possible, employing the main housing containing the electronics of the instrument to which they belong.

- Sensors should be subjected to an "as received" evaluation of their performances prior to adjustment. The information thus obtained could be useful for adjusting already collected data to account for sensor drift or errors during deployment.

- It may be useful to occasionally employ a calibration service provider different from the usual one; for example, if calibrations are routinely performed inhouse, they could be done externally every once in a while. Over time, this custom will provide information useful for quality assurance.

- Calibration records must be kept up-to-date; calibration histories of sensors can often help to pre-empt potential problems with them in time.

- The results of a calibration may or may not be accredited but they must always be accompanied by the following:

- A declaration of the uncertainty associated with the calibration process;

○ Information evidencing traceability to reference material (certified or otherwise).

\section{b) Towards a European Marine Calibration Network}

As revealed through the JERICO calibration activities and in particular Deliverable 4.1 "Report on existing calibration facilities", very few observatory operators actually maintain dedicated calibration facilities with trained personnel. Thus very often sensors are shipped to manufacturers on regular basis which is neither convenient nor cost efficient as mentioned before. Partners operating calibration facilities often face difficulties in maintaining dedicated personnel positions as funding is variable and rather insecure. Although there is significant experience among European research institutes on calibration methods, at present each lab works independently with no or very little connections with other labs.

Acknowledging the above issues JERICO has promoted the establishment of a permanent working group for calibration activities, proposing a future strategic plan towards a permanent, pan-European calibration grid to support the activities of marine observatories. The grid will be open to the whole marine community and in close connection with the national metrological institutes while in order to maximise benefits and minimise costs it can have a 2 level approach (figure 1), separating calibration procedures into primary and secondary. In the first level, labs capable of handling reference calibration procedures will be identified and appointed as Primary Reference Nodes (PRN) where secondary calibration instruments can be calibrated. Level 2 or Secondary Reference Nodes (SRN) will use the secondary reference instruments calibrated at PRN and will be responsible for the calibration of the day-to-day operational sensors around European Waters (Figure 2). 


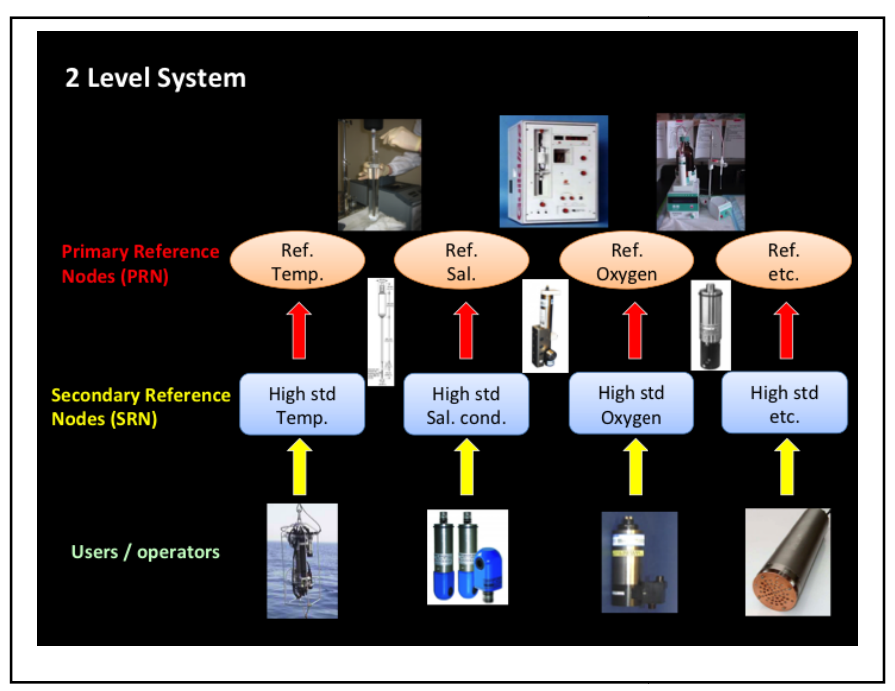

Fig. 1. The 2 level approach

Building this grid will require fostering cooperation between people and groups to promote knowledge exchange and training, nurturing consensus on methodologies and procedures, harmonizing standards of operation, achieving Best Practice, and a rational coordination of resources.

To accomplish the above it is important to find an appropriate funding mechanism within EU while a rational roadmap will involve 2 stages. During the first stage the consortium will be set up with actions focusing on documenting of what is in place, identification of PRN and SRN, harmonisation and standardisation activities. In the second stage funding efforts will be concentrated towards strengthening the existing infrastructures considering that although the calibration procedures of Temperature and Salinity are well defined, there is a dynamic evolution in terms of biogeochemical sensors. Thus labs have to be equipped with state of the art instruments to perform state of the art calibrations and at the same time to carry experiments for developing new methods.

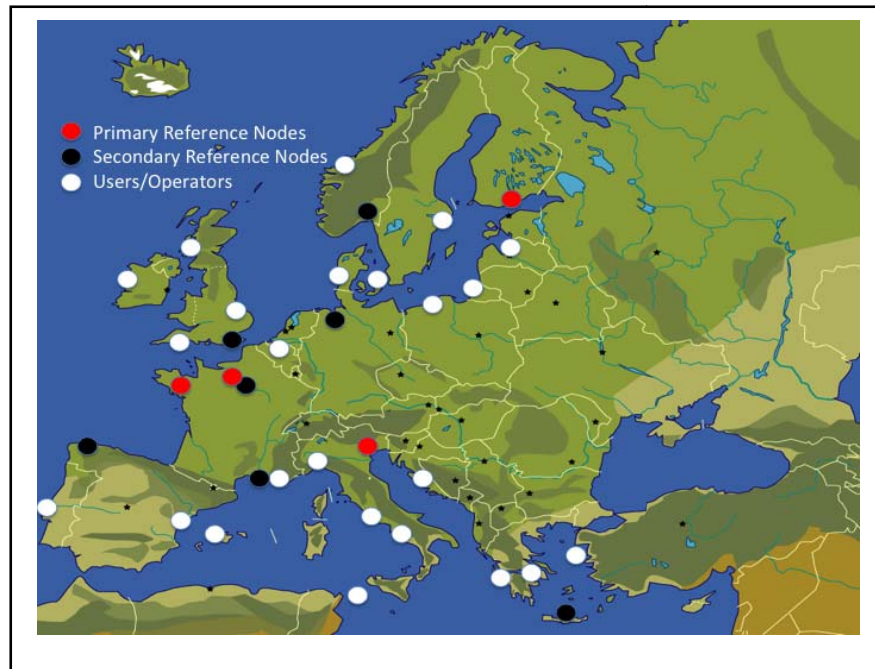

Fig. 2. . The European distribution of calibration nodes and users/operators

\section{Costs}

The cost of setting up and operating such systems can be significant including scheduled and unforeseen expenses including routine operation, repair and replacement of equipment, personnel costs and accidents. Observing systems within Europe have been funded through a variety of national and EU programs. These have frequently been programs, which fund observatories for fixed periods of time rather than providing funding for sustained observations. An analysis of the running costs of observing systems was undertaken in order that better-informed decisions about their sustainability can be made. The complexity of these systems varies in both the types of parameters measured (physical, chemical, biological) and the nature of the platform (towers, pylons, moorings, research vessels, ships of opportunity, gliders).

JERICO provided an opportunity to describe in an analytical form the expenses emanating from the operation of each different system (fixed platforms, FerryBox, gliders) and calibration laboratories. This is a valuable tool to enable the operators to compare, adjust, improve and exchange practices with the ultimate goal of minimizing costs and maximizing the scientific value of the infrastructure.

Information for the analysis of costs was gathered using a questionnaire that was designed in February 2012 at the Rome JERICO workshop and modified in discussions with GROOM participants. A joint JERICO/GROOM - EGO Glider Workshop was held on 22-23 May 2012 in Mallorca during which costs for operating a glider fleet were assessed by each participating institution.

The average initial investment and annual running costs for fixed platforms, FerryBox systems and calibration laboratories in Europe were considered based on the results of the questionnaire. There was a large variability in costs between laboratories reflecting the different types of platforms and parameters being measured. However, the figures give an indication as to the level of investment required and annual running costs for fixed platforms, FerryBox systems and calibration laboratories. Initial investment costs are greater for glider fleets $(€ 222,545$ in 2011) and FerryBox systems $(€ 110,298$, min $€ 20,600$, $\max € 259,105)$ than for fixed platforms $(€ 86,526$, min $€ 1000$, max $€ 334,000)$. Ongoing total annual running costs for a glider fleet ( $€ 184,014$ excluding investment in 2011) and fixed platforms (€139,358, min $€ 35,217$, max $€ 307,500)$ exceed those of FerryBox systems $(€ 90,529$, min $€ 19,550 \max € 151,250)$. Personnel costs account for $44 \%, 49 \%$ and $55 \%$ respectively of the total annual running cost of a glider fleet $(€ 81,430)$, fixed platforms $(€ 68,615)$ and FerryBox systems $(€ 49,565)$. The analysis of costs has shown that a large proportion $(27 \%)$ of the total annual running cost of fixed platforms is associated with boat charter $(€ 37,315)$. Collaborative working such as under the Eurofleets project (http://www.eurofleets.eu/np4/63) may give the opportunity to reduce these costs and maximize efficiency.

\section{Best Practices - JERICO Label}

As already mentioned coastal observations are an important part of the marine research puzzle of activities and 
applications. However significant heterogeneity exists in Europe concerning technological design of observing systems, measured parameters, practices for maintenance and quality control, as well as quality standards for sensors and data exchange. An important task of JERICO was to describe best practices in all phases of the system; to adopt common methodologies and protocols and to move towards the harmonization of equipment, which will help in reducing maintenance and costs. A key tool identified right from the start of the project was the value of workshops and common exercises. Considering that an important aspect of a project like JERICO is to bring together the uncoordinated community, seeking cooperation, exchange of expertise and knowledge, face-to-face meetings proved extremely valuable towards this. Thus although dedicated funds for such activities were not originally reserved, in close cooperation with WP3 a number of workshops and exercises were organised taking advantage of scheduled meetings - back to back with GA - and international events (conferences, workshops, shows, exhibitions etc.).

In the four years of the project six workshops were organised with a higher frequency in the first two years, as there were a lot of issues/actions that partners had to discuss, agree and schedule. Harmonisation proved particularly difficult since as described in the D4.4 Report on Best Practice in Conducting Operations and Maintaining, the harmonisation and Best Practices needs, greatly depend on the platform. Thus Gliders are the platform where a lot of work was already in place for three main reasons:

- There is relatively very small variability among the different types of Gliders in the market. This is because custom made Gliders cannot be build and all the users rely on market products.

- All available products are built upon the same principle and utilise very similar technology

- Activities during GROOM project funded very early in the life of Gliders significantly contributed in bringing together the Gliders community. In this framework operators formed a coherent group inside which, practices and experiences were exchanged.

In the case of FerryBox, although there is room for custom designs, since the system is designed for ships monitoring only the surface ocean, the amount of customisation is limited. As with Gliders the FerryBox community in the very early steps benefited from the FerryBox FP5 project [6] and judging from the work described in the deliverables and the strong connection between partners remained in the years after, it has been a key step. Although variations on the standard FerryBox approach have been done in the last years (sailing and fishing boats) the practices and protocols largely remain the same.

Unfortunately Fixed Platforms as expected exhibit the highest variability with many different designs across Europe, employing significantly variable techniques of operation and maintenance. Great variability is also found both in the observing methods as well as in the part of the environment monitored. The main reasons for the great variation found are:
- Many different designs produced both as off the shelf products as well as custom builds.

- In most cases, designs follow a fit-for-purpose approach adopted for the environment in which they are placed

- The environmental constraints in the coastal environment are high

- The variability of sensors that can be placed on board Fixed Platforms is very high

Irrespectively of the above problems Best Practices for all three coastal platforms were defined and documented in what we think will be a reference document for the future.

In addition to the above the JERICO partners introduced the concept of a JERICO Label in order to:

- Acknowledge a consensus on guidelines for best practices in the design, the implementation, the maintenance, the data policy and the valorisation of operational coastal observatories;

- Achieve fair recognition of the quality of the managed observatories for the partners and all new comers that comply with this Label;

- Help stakeholders becoming aware of the European interest in the development of high quality coastal observatories;

- Foster a wider market for the industry in sensor technology and platforms based on the agreed recommendations.

The JERICO Label is a set of criteria defined to ensure some standardisation and interoperability, and the quality of data for coastal observatories. Observation systems certified by the JERICO Label will be internationally recognised. More specifically:

\section{1) Sustainability}

The sustainability is a critical issue for each infrastructure/platform especially in the coastal area where funding is limited to national funds.

- Although financing of coastal observation systems is often variable, a 5-year funding road map from National and/or International sources is required.

- Long time-series and, when possible, synopticity of the integrated multi-platform observing platforms is considered as an advantage.

\section{2) Operationality}

A key aspect of an efficient operational observing system is the streamlined processing of real time and delayed mode data, which takes the data from raw to quality assured. Considering the money and effort invested at EU level through numerous projects and initiatives such as SeaDataNet, EuroGOOS 
Regional Centres and MyOcean, the observation system must ensure that the flow of real-time and delayed mode data will be reliable, accessible and easy to distribute. To do so there are number of key issues which must be considered:

- Data is quality controlled following documented protocols.

- Free and open access according to Aarhus Convention on environmental data as expressed by IOC Data Policy (International oceanographic Commission UNESCO).

- Long term archiving (more than 20 years) policy and implementation has to be performed for all types of data, including classified data. Archived datasets should be citable with a mention of the observation system.

- Clear mechanism must be in place to guarantee data authorship traceability.

- Data availability (real time/near real time - delayed mode) compatible with the "observation method" (for example real time is required in operational systems).

- Data frequency is compatible with the "observation purpose" capturing the time scale(s) of the observed phenomena.

In particular the JERICO Label will be in compliance with the specification of the Group on Earth Observations (GEO) Label. The main goal of the Label is to specify an approach on how to judge the quality of data, based on the completeness of information as to how the data have been acquired, i.e. completeness of metadata description.

\section{3) Observing purpose:}

Considering the big variability between coastal observatories and the peculiarities of the coastal environment a "Fit for Purpose" approach has been chosen. Thus in terms of measured parameters the observation system must have:

- Primary or Core Parameters. These are basic parameters required for the specific observing purpose following the UK Integrated Marine Observing Network Initiative (UK-IMON) core parameter list (http://www.jericofp7.eu/deliverables/d14-label-definition).

- Secondary Parameters. These are additional parameters which are 'good to have' and although don't fall within the "Primary or Core" category, they are also measured.

An integral part of the Label is the extensive list of Recommendations emanating from the Best Practices defined covering all-important aspects in terms of deployment, operation and maintenance.

\section{ACKNOWLEDGMENT}

The work was supported by the FP7INFRASTRUCTURES-2010-1, Towards a Joint European Research Infrastructure Network for Coastal Observatories JERICO, Grant Agreement Number 262584, coordinated by IFREMER.

\section{REFERENCES}

[1] Petersen, W., et al., FerryBox: From "On-line Oceanographic Observations to Environmental Information"., in EuroGOOS Publication No. 25., E. Office, Editor. 2007: SHMI, 60176 Norkoepping, Sweden.

[2] Nittis, K., et al., "The M3A multi-sensor buoy network of the Mediterranean Sea". Ocean Science, 2007. 3: p. 229-243.

[3] Petihakis, G., et al., "M3A system (2000 - 2005) - operation and maintenance", Ocean Science, 2007. 3: p. 117-128.

[4] Delauney L. and C. Compère., "Biofouling protection for marine environmental sensors by local chlorination", in Springer Series on Biofilms, Marine and Industrial Biofouling, Springer, Editor. 2009. p. 119-134.

[5] Faimali, M., et al., "The interplay of substrate nature and biofilm formation in regulating Balanus amphitrite Darwin, 1854 larval settlement", Journal of Marine Biology and Ecology, 2004: p. 37-50.

[6] Petersen, W., "FerryBox Systems: State-of-the-Art in Europe and Future Development". Journal of Marine Systems, 2014. 140: p. 4-12. 